\title{
LIDERAZGO DIRECTIVO Y CAMBIO EDUCATIVO: ANÁLISIS DE UNA EXPERIENCIA DE COLABORACIÓN UNIVERSIDAD-ESCUELA
}

\author{
Directive Leadership and Educational Change: An Analysis of a Collective \\ School-University Experience
}

\author{
Carolina Aparicio Molina ${ }^{1}$ \\ ORCID: 0000-0003-3505-5725 \\ Correo: caparicio@ucsc.cl \\ Felipe Sepúlveda López ${ }^{2}$ \\ ORCID: 0000-0001-5703-383X \\ Correo: fsepulveda@ucsc.cl \\ Ximena Valverde Huincatripay ${ }^{3}$ \\ ORCID: 0000-0002-6010-8051 \\ Correo:ximval@gmail.com \\ Víctor Cárdenas Merino ${ }^{4}$ \\ ORCID: 0000-0001-6362-0344 \\ Correo: vcardenas@coalco.cl \\ Gladys Contreras Sanzana ${ }^{5}$ \\ ORCID: 0000-0001-8244-1405 \\ Correo: gcontreras@ucsc.cl \\ Marcela Valenzuela Ravanal ${ }^{6}$ \\ ORCID: 0000-0003-1984-8506 \\ Correo: mvalenzuela@ucsc.cl \\ ${ }^{1256}$ Universidad Católica de la Santísima Concepción, Chile. \\ ${ }^{34}$ Colegio Alerce, Concepción, Chile.
}

Recibido: 04/12/2018

Aceptado: 02/03/2020

Resumen: El propósito de este artículo es analizar las implicancias de un Plan de Trabajo, diseñado por el equipo de investigación, para orientar procesos de mejora que se estaban desarrollando en el equipo directivo de un establecimiento educacional integrante de una Red de Colaboración Universidad-Escuela. En el plan se consideraron fases basadas en la revisión del Modelo de Liderazgo Instruccional y el fortalecimiento de las competencias de liderazgo a partir de las comunidades profesionales de aprendizaje. Se siguió un diseño metodológico correspondiente a un estudio descriptivo de casos de método mixto y transformador. Los resultados evidenciaron que el plan fue una experiencia de acompañamiento que facilitó al equipo directivo una real orientación al cambio y al desarrollo de capacidades para dar respuesta a las exigencias propias de la institución educativa que dirigían.

Palabras clave: liderazgo directivo; cambio educativo; liderazgo instruccional; comunidades profesionales de aprendizaje. 
Abstract: The purpose of this article is to analyze the implications of a Work Plan, designed by the research team, to guide improvement processes that were being developed in the management team of an educational establishment that is part of a University-School Collaboration Network. The plan considered phases based on the revision of the instructional leadership model and the strengthening of leadership competencies based on professional learning communities. A methodological design was followed corresponding to a descriptive study of cases of mixed and transforming method. The results showed that the plan was an experience of accompaniment that provided the management team with a real orientation to change and to the development of capacities to respond to the demands of the educational institution that they directed.

Keywords: directive leadership; educational change; instructional leadership; professional learning communities.

\section{Introducción}

La investigación que se presenta a continuación ${ }^{1}$ se desarrolló en el marco de una Red de Colaboración de la Universidad Católica de la Santísima Concepción (RC-UCSC) — Región del Biobío, Chile - con siete escuelas de la ciudad. Su objetivo fue analizar las implicancias de un Plan de Trabajo que se diseñó con el propósito de fortalecer procesos de mejora impulsados por el equipo directivo de uno de los establecimientos educacionales integrantes de esta red.

La RC-UCSC surgió en el marco del Plan de Mejoramiento Institucional (2015-2018) denominado "Propuesta de mejoramiento para provocar cambios notables en los procesos de formación inicial docente en la UCSC, con énfasis en el trabajo colaborativo con actores del sistema educativo regional en contextos educativos diversos" (PMI1501) financiado por el Ministerio de Educación de Chile. Bajo este contexto, el equipo de investigación buscó facilitar procesos de investigación educativa relacionados con las necesidades de las comunidades escolares de dicha red que a su vez generaran conocimiento relevante para mejorar la formación inicial docente. Por ello, y en vista que las comunidades educativas presentan diversas áreas en las cuales es importante y valioso generar conocimiento, el equipo optó por el área de liderazgo directivo.

El liderazgo directivo es un ámbito que, como lo indica el Informe TALIS (Organisation for Economic Cooperation and Development, 2019), puede mejorar sustantivamente las condiciones laborales y de aprendizaje de los profesores, proceso que impacta en los aprendizajes

\footnotetext{
${ }^{1}$ La investigación fue financiada por la Facultad de Educación de la Universidad Católica de la Santísima Concepción a través del proyecto NI. EDUC 032018 y la Dirección de Investigación de la misma Universidad por medio del proyecto DIN 18/2016.
} 
de los estudiantes. Pero en Chile, como en muchos países, se ha visto la necesidad de fortalecer las prácticas de liderazgo en tanto se ha evidenciado una distancia entre el liderazgo y las prácticas instruccionales, es decir las focalizadas en los procesos de enseñanza aprendizaje (Sepúlveda y Aparicio, 2019). Esta necesidad es además requerida en tanto, a nivel latinoamericano, los datos delatan una escasa producción científica sobre el liderazgo directivo, siendo el porcentaje de artículos publicados en América Latina en torno al liderazgo no superior al 1\% (Aravena y Hallinger, 2018). En este sentido, Hallinger (2019) señala que en Chile existe un escaso desarrollo en investigación sobre el liderazgo educativo. Por su parte, Weistein et al. (2019) realizaron una revisión sistemática de artículos sobre esta temática entre los años 2008 y 2019 y llegaron a la conclusión que en Chile la investigación en esta área se encuentra en una fase emergente, a pesar de que a partir del 2016 se genera un aumento significativo de artículos.

De esta manera, es necesario analizar y generar conocimiento sobre el liderazgo directivo y las implicancias de los cambios que, durante los últimos 13 años, se han observado a nivel de políticas; especialmente sobre la tendencia a fortalecer un modelo de liderazgo educativo que supone un trabajo integral y focalizado en prácticas efectivas para cada contexto escolar (Ahumada, Galdames, y Clarke, 2016; Cuellar y Giles, 2012; Flessa, 2014; Tapia-Gutiérrez, Becerra-Peña, Mansilla-Sepúlveda, y Saavedra-Muñoz, 2011; Quiroga, y Aravena, 2018; Ulloa Garrido, Nail Kröyer, Castro Hidalgo y Muñoz Reyes, 2012; Weinstein y Hernández Vejar, 2014).

Este modelo de liderazgo directivo -impulsado especialmente a través de una compleja política de fortalecimiento de capacidades directivas y orientado por un documento titulado Marco para la Buena Dirección y Liderazgo Escolar (Ministerio de Educación de Chile, 2015) — estimula la reflexión y planificación de estrategias por parte de los líderes escolares, que facilitan la innovación y la mejora de resultados en distintos indicadores de calidad educativa. Junto a lo anterior, las normativas nacionales (entre ellas Ley 20.248, Ley 20.903 y Ley 20.845) han tendido a dotar al director/a y al equipo directivo de la responsabilidad de orientar las prácticas pedagógicas, fortalecer las competencias y los conocimientos requeridos por los docentes y miembros del equipo de gestión en la implementación del liderazgo escolar. Estas responsabilidades de los cargos se han instaurado a través del marco legal sin evaluar capacidades y esfuerzos de los equipos de trabajo de acuerdo con los contextos en los cuales se desempeñan.

En consecuencia, si bien los documentos que prescriben el trabajo directivo debiesen haber sido un marco facilitador del desarrollo de la escuela a la vez que profesional de los directivos, no 
ha facilitado el desarrollo de capacidades para el mejoramiento. La evidencia indica que los procesos de mejora educativa en Chile se han establecido en contextos regulados principalmente bajo modelos de rendición de cuentas, modelo que a nivel de gestión escolar tensiona y limita el desarrollo interno de la escuela, en tanto predominaría en los líderes la búsqueda de respuestas a exigencias externas de carácter centralista en detrimento del desarrollo profesional de los educadores (Flores, 2014; Hargreaves y Shirley, 2012; Falabella y de la Vega, 2016).

De esta manera, las orientaciones y normativas se encontrarían distantes de la práctica del liderazgo en la escuela y especialmente del componente pedagógico del liderazgo directivo. Este desafío demanda a la investigación educativa conocer y comprender dichas prácticas para apoyar su mejoramiento. Esto impacta en la percepción de logro en los equipos directivos, y en la sociedad en general, respecto a la capacidad de estas escuelas para generar mejoras en los aprendizajes de los estudiantes. Esta percepción se traduce en una constante reducción de matrículas y disminución de la valoración social de la escuela que atiende a niños y jóvenes en situación de vulnerabilidad socioeconómica (Horn y Marfán, 2010; Ruffinelli, 2016; Valenzuela y Montecinos, 2017).

Sumado a esta tensión, ya se había demostrado que en Chile gran parte del mejoramiento de los resultados de aprendizaje en escuelas habrían sido orientados por un sentido de liderazgo que buscaría la eficacia escolar. Esto se traduce en un trabajo pedagógico dirigido a mejorar los resultados de aprendizaje medidos a nivel estandarizado en el contexto nacional para, en un futuro próximo, potenciar los logros más allá de lo esperado por las condiciones de vulnerabilidad asociada a los estudiantes (Horn y Marfan, 2010). Este es un antecedente que, a la luz de la realidad, cuestiona los usos de la medición estandarizada nacional y su potencial para orientar procesos de mejora.

A la luz de estos antecedentes, es necesario indicar que los establecimientos que forman parte de la RC- UCSC adhieren a la Ley 20.248 de Subvención Escolar Preferencial (SEP). Esta ley hace entrega de una subvención económica diferenciada de acuerdo con el porcentaje de estudiantes matriculados que forman parte del grupo socioeconómicamente más vulnerable del país (alumnos preferentes). Este dinero se entrega a la vez que se exige el diseño, implementación y evaluación de un Plan de Mejoramiento orientado a alcanzar resultados más altos en indicadores nacionales de aprendizaje. Este plan debe presentar evidencias concretas sobre los logros alcanzados. 
A partir de lo expuesto, como equipo de investigación y junto a los directivos de los establecimientos de la RC-UCSC, comprendimos que debían existir dificultades en la gestión del liderazgo directivo de dichos establecimientos educativos que no les eran exclusivas, sino parte de una problemática nacional. Así, surgió como problema que frente a la tendencia a aspirar al logro de la equidad educativa por medio de normativas a nivel país, no se fortalecería la autonomía en el liderazgo de las escuelas y necesariamente la investigación educativa debiese avanzar en propuestas de cambio más que en evidenciar necesidades ya descritas en otros estudios.

Es así como se planteó avanzar en experiencias de investigación situada que lograran cambios en las prácticas directivas a través de un proceso complejo que reconociera a los directivos como actores fundamentales del cambio educativo y, por tanto, de cualquier innovación en esta área. Para ello se optó, en primer lugar, por seguir la propuesta del Modelo de Liderazgo Instruccional para conocer fortalezas y debilidades en esta área de los equipos directivos de la RCUCSC. Este modelo es reconocido y ha inspirado documentos institucionales como el propio Marco para la Buena Dirección, además de resultar un modelo aplicable en Chile (Day et al., 2009; Fromm, Olbrich y Volante, 2015). Por este motivo se aplicó el cuestionario PIMRS a 205 docentes de la RC-UCSC, tanto a equipos directivos como docentes de los siete colegios.

En segundo lugar, se consideró que, a fin de efectuar procesos de investigación que verdaderamente dieran cuenta de una Red de Colaboración entre la Universidad y la Escuela, era necesario fortalecer el liderazgo educativo en las competencias profesionales al interior de los centros. Como han planteado Hargreaves y Fullan (2014) se requería conocer y dialogar con el capital profesional de los centros. De este modo, se consideró el desarrollo de un Plan de trabajo para uno de los establecimientos de la RC-UCSC a partir de las comunidades profesionales de aprendizaje (CPA). Esta propuesta es referenciada por investigadores como Darling-Hammond et al. (2017) por su potencial para aportar a la mejora del clima organizacional y la cultura escolar, lo que a su vez resulta relevante en el mejoramiento de los resultados de aprendizaje (Fullan, 2012).

Las CPA son una vía de puesta en práctica del liderazgo distribuido y la gestión de escuelas en los márgenes del aprendizaje organizativo y del trabajo colaborativo (Harris, 2012). Pero, principalmente, son un medio para alcanzar una cultura de colaboración y de responsabilidad colectiva que ha demostrado mejorar el aprendizaje de los estudiantes y también de los docentes. Estos logros son atribuidos al enfoque en el trabajo colaborativo comprometido con un propósito común, como mejorar las prácticas docentes y cuidar el desarrollo profesional. Por ello, las CPA 
además de buscar la mejora de los aprendizajes de los estudiantes buscan alcanzar estos fines a partir de la construcción de un criterio colectivo, de un sentido compartido del quehacer docente (Hargreaves y Fullan, 2014, p.158).

Las competencias profesionales han demostrado ser relevantes para la mejora sistemática de la calidad de la educación, especialmente en lo que respecta a la percepción de los profesores de que trabajan para generar aprendizajes valiosos y que a su vez están mejorando su propio desarrollo profesional, percepción que finalmente afecta significativamente en la mejora de los aprendizajes de los estudiantes (Klieger, 2016; López y Gallegos, 2014; Muñoz Martínez y Garay Garay, 2015; Tanriogen e Iscan, 2016).

En consecuencia, se reporta en esta comunicación el proceso de desarrollo de un Plan de Trabajo diseñado por el equipo de investigación a partir de los antecedentes teóricos descritos (Liderazgo Instruccional y CPA), considerando principalmente las implicancias que tuvo su implementación para los directivos. De esta manera, el análisis del Plan es una tarea relevante para conocer sus fortalezas, alcances y potencialidades de continuar trabajando en esta línea.

\section{Metodología}

El diseño metodológico correspondió al de un estudio descriptivo de casos (Yin, 1993) por medio de un método mixto donde se integraron estrategias de generación de datos cuantitativos y cualitativos, en el diseño denominado transformador (Creswell, 2012, p. 546). Este diseño considera un complejo marco en el cual se orienta el trabajo de investigación a hacer aportes en temas de alto interés social, como en este caso es el liderazgo educativo en contextos de población vulnerable.

A raíz del diseño escogido, se utilizó como método de selección de casos al sondeo opinático descrito por Vieytes (2004), que permitió involucrar a los siete establecimientos educativos que formaban parte de la Red de Colaboración UCSC, para una primera etapa, y un caso (Colegio 1) para el desarrollo de la segunda etapa. Al ser la segunda etapa la de mayor énfasis transformador, se buscó la selección de un caso relevante. El caso fue elegido por su disposición para participar en un Plan de trabajo en red entre directivos y académicos universitarios, así como también por presentar indicadores de Liderazgo Instruccional reducidos en comparación con el grupo completo. El caso se describe a continuación: 
El Colegio 1 posee las características de un establecimiento educacional científicohumanista y técnico-profesional, ubicado en un sector urbano de la Ciudad de Concepción. Fue fundado el año 2009 por medio de una entidad sin fines de lucro, quien a la fecha ejerce como sostenedora. Tiene una matrícula de 312 estudiantes, desde $1^{\circ}$ año de Enseñanza Básica a $4^{\circ}$ año de Enseñanza Media, y 30 profesores. De acuerdo con la normativa vigente en Chile, el Colegio 1 es gratuito, no realiza selección a los estudiantes, está adscrito al proceso nacional de inscripción y cuenta con Proyecto de Integración Escolar para atender con diferentes profesionales a estudiantes que presenten necesidades educativas especiales.

Los integrantes del Colegio 1 que participaron del proyecto de investigación conocieron en todo momento los fines y métodos de trabajo y accedieron voluntariamente a formar parte del proceso por medio del asentimiento y la firma de consentimientos informados.

Las actividades de investigación derivadas de este diseño fueron las siguientes:

\section{Etapa I. Diagnóstico de habilidades de liderazgo instruccional desarrolladas por parte de los equipos de gestión}

1) Aplicación del cuestionario PIMRS de Liderazgo Instruccional: El cuestionario para evaluar las habilidades de liderazgo instruccional utilizado correspondió a la "Escala de Percepción de la Gestión Instruccional de Directores" (Principal Instructional Management Rating Scale, PIMRS) elaborado por Hallinger y Murphy (1985). Este instrumento ha sido utilizado para el desarrollo de estudios empíricos en reconocidas investigaciones en Chile y en diferentes sistemas escolares (Hallinger, 2011). El modelo propone tres dimensiones vinculadas al ejercicio del liderazgo instruccional y cada dimensión se analiza en la implementación de 10 acciones a ser implementadas por el directivo o líder escolar. La primera dimensión es Definir la misión de la escuela, y corresponde al rol del director garantizar que la escuela presenta una misión clara, conocida y orientada al progreso académico de los estudiantes. La segunda dimensión es Administrar el plan académico de la escuela, que aborda las acciones ligadas al control y seguimiento del plan académico del colegio por parte del director, enfatizando con ello las capacidades de delegar y compartir la responsabilidad con los especialistas. La tercera dimensión, Fomento de un adecuado clima de aprendizaje escolar, describe acciones que abordan la exigencia académica y las expectativas que se tienen en torno al desarrollo de los profesores y su capacidad de aprender en la escuela. 
El PIMRS es en definitiva una encuesta de 50 preguntas que evalúan la frecuencia en que cada director demuestra el desarrollo de las acciones asociadas al liderazgo instruccional. Cada pregunta se responde por medio de una escala de tipo Likert que varía de casi nunca (1) a casi siempre (5). Se aplica una forma que la responde el director y otra forma es completada por los docentes del establecimiento. El estudio original de validación del instrumento encontró que el PIMRS cumple con altos estándares de confiabilidad, ya que cada una de las 10 subescalas evaluadas excedieron .80 en el test de consistencia interna de Cronbach (Hallinger, 2012).

2) Sistematización de los datos relevados: Los resultados del instrumento PIMRS aplicado a 183 docentes y 22 directivos (N 205), pertenecientes a los siete establecimientos educacionales de la Red UCSC, fueron sistematizados en una planilla de MS Excel para luego realizar un análisis de frecuencia. Considerando la información de toda la muestra participante se examinaron las disparidades en la percepción de los equipos de gestión con respecto a los equipos docentes, relativos a la implementación de las funciones directivas consideradas en el modelo de desarrollo de prácticas de liderazgo instruccional. Este primer análisis permitió tener una visión general respecto a las fortalezas y debilidades en el desarrollo de prácticas de liderazgo instruccional de todos los establecimientos educativos que forman parte de la RC-UCSC. Los resultados obtenidos fueron presentados en una jornada de trabajo donde a cada colegio se le entregó un reporte que consideró una contextualización del Liderazgo Instruccional y orientaciones para la mejora de acuerdo con los resultados obtenidos.

\section{Etapa II. Surgimiento de propuesta de innovación en la gestión y liderazgo}

3) Selección de un establecimiento para generar una propuesta innovadora: Tras la entrega de los informes, se invitó al equipo directivo del establecimiento seleccionado (Colegio 1) a participar en un proceso consistente en analizar los resultados obtenidos en el PIMRS y, a partir de la propuesta de las comunidades profesionales de aprendizaje (Roberts \& Pruitt, 2009), diseñar un plan de trabajo que permitiera abordar la complejidad de la tarea directiva. La metodología de las CPA corresponde a un esfuerzo dirigido y delimitado a la mejora de los resultados de aprendizaje de los estudiantes a partir del encuentro profesional entre los docentes. El equipo directivo, por medio de su encuentro y discusión permanente, analizó el modelo y determinó las prácticas que en su contexto permitían mejorar los resultados de los estudiantes, potenciar el desarrollo profesional de los docentes y su propio desempeño como equipo directivo. 
4) Diseño e implementación de la propuesta: El trabajo se efectuó a través de reuniones periódicas durante el año 2017 entre los investigadores y miembros del equipo de gestión del Colegio 1, para elaborar e implementar un Plan de Trabajo. Este plan consideró elementos centrales de todo proceso de gestión, es decir: acciones, involucrados o responsables y evaluación, pero para dar valor a la propuesta teórica se incorporaron conceptos propios del Liderazgo Instruccional como las dimensiones y acciones respectivas. También, bajo los supuestos teóricos planteados desde la academia, se establecieron Focos de las CPA, a fin de que directivos y académicos estuvieran en sintonía sobre los elementos en común de ambas miradas teóricas. Igualmente se incorporó la idea de una frecuencia orientadora para quienes implementaban el plan de trabajo.

Las reuniones fueron coordinadas por los investigadores para ser desarrolladas a los menos una vez por mes en el establecimiento educativo. Igualmente se generaron encuentros a solicitud de los participantes en otros momentos del año y en espacios como la propia universidad.

\section{Etapa III. Evaluación del alcance de la propuesta trabajada}

5) Evaluación de resultados a través de la reaplicación de cuestionarios y entrevistas semiestructuradas: Una vez finalizada la aplicación de los planes se realizó una reaplicación de una dimensión del cuestionario PIMRS para evaluar el impacto del plan en los profesores y miembros del equipo directivo. Junto a lo anterior, al inicio del curso lectivo año 2018, se desarrollaron entrevistas semiestructuradas (Coffey y Atkinson, 2003) a los miembros del equipo directivo que participaron de la innovación. Los entrevistados fueron el director y la encargada de evaluación, quienes firmaron un consentimiento informado. Las entrevistas fueron registradas en audio, transcritas y analizadas a través de la técnica de Análisis de Contenido (Marradi, Archenti y Piovani, 2018, p. 421).

\section{Resultados}

Los resultados obtenidos permitieron comprender la relevancia de la adecuada integración de los métodos de investigación para generar un cambio educativo potencialmente sostenible en el tiempo (Creswell, 2012; Fullan, 2012). Los resultados se presentan siguiendo las etapas desarrolladas: 


\section{Etapa I. Diagnóstico de habilidades de liderazgo instruccional desarrolladas por parte de los equipos de gestión}

La compilación de las respuestas recogidas en los siete colegios participantes de la Red UCSC permitió observar tendencias generales de las prácticas de gestión y liderazgo asociadas a las tres dimensiones del liderazgo instruccional (Figura 1). Los siete equipos directivos de la Red se perciben a sí mismos cumpliendo con los requerimientos que han sido enfatizados en documentos como el Marco para la Buena Dirección y Liderazgo Escolar (Ministerio de Educación de Chile, 2015). Los ámbitos de interés de este marco son coincidentemente dimensiones en las que los directivos indican trabajar y poner sus esfuerzos para efectuar mejoras, como se evidencia en las dimensiones I y III: Define la misión de la escuela y Fomenta un clima adecuado para el aprendizaje.



Figura 1. Resultados de los equipos directivos y docentes de los siete establecimientos que forman parte de la Red UCSC con respecto a las tres dimensiones de liderazgo instruccional (N 205).

Fuente: Elaboración propia.

A su vez, la Figura 1 revela que las tareas de la dimensión II, Administra el plan académico de la escuela, no forman parte de las que mayormente realizan los directivos, tanto desde la percepción de los propios equipos directivos como de los docentes. Esto se entiende porque en Chile la figura de la Unidad Técnico-Pedagógica (UTP), con uno o más profesionales encargados de atender el ámbito curricular en los establecimientos educativos, cumple principalmente con el rol asociado a las tareas de esta dimensión. 
Por último, en la gráfica (Figura 1) se observa que la mayor disparidad entre las percepciones de los equipos directivos y los docentes encuestados se aprecia en los resultados de la dimensión III. Esto sugiere que los equipos directivos no realizarían esfuerzos relevantes, según el profesorado, para el desarrollo de capacidades en los docentes y la creación de ambientes que fomenten y valoren el aprendizaje de todos los miembros de la comunidad escolar.

\section{Etapa II. de Surgimiento de propuesta de innovación en la gestión y liderazgo}

Las tendencias generales obtenidas en la Etapa I se utilizaron como base empírica para el diseño de la Etapa II, en la que participó en Colegio 1. Como se mencionó, esta institución deseaba participar y sus resultados cumplían con la característica de ser disminuidos en relación al grupo completo, especialmente en las disparidades entre docentes y directivos.

La información obtenida en la Etapa I fue analizada de forma detallada con el director y la encargada de evaluación del Colegio 1. Específicamente se examinaron los resultados de cada una de las 10 funciones directivas consideradas en el cuestionario de Liderazgo Instruccional y aplicado a 33 docentes y 5 miembros del equipo de gestión del Colegio 1: director, jefe de la UTP, coordinadora de currículum y evaluación y coordinadores de ciclo (Figura 2).

Los resultados generados tras el análisis de frecuencia de las respuestas dadas en el margen de una escala de Likert y agrupados por estamento reflejaron una amplia disparidad entre la percepción de los docentes y director respecto a cómo este último desempeñaba las acciones asociadas a cada función. Especialmente esta disparidad se evidencia para las acciones directivas asociadas a la Definición de las metas de la escuela y Fomento de un adecuado clima de aprendizaje escolar, ámbito en el que por ejemplo "Provee incentivos para profesores", "Promueve el desarrollo profesional" y "Provee incentivos para el aprendizaje" demostraron una percepción del director y de los docentes opuesta. 


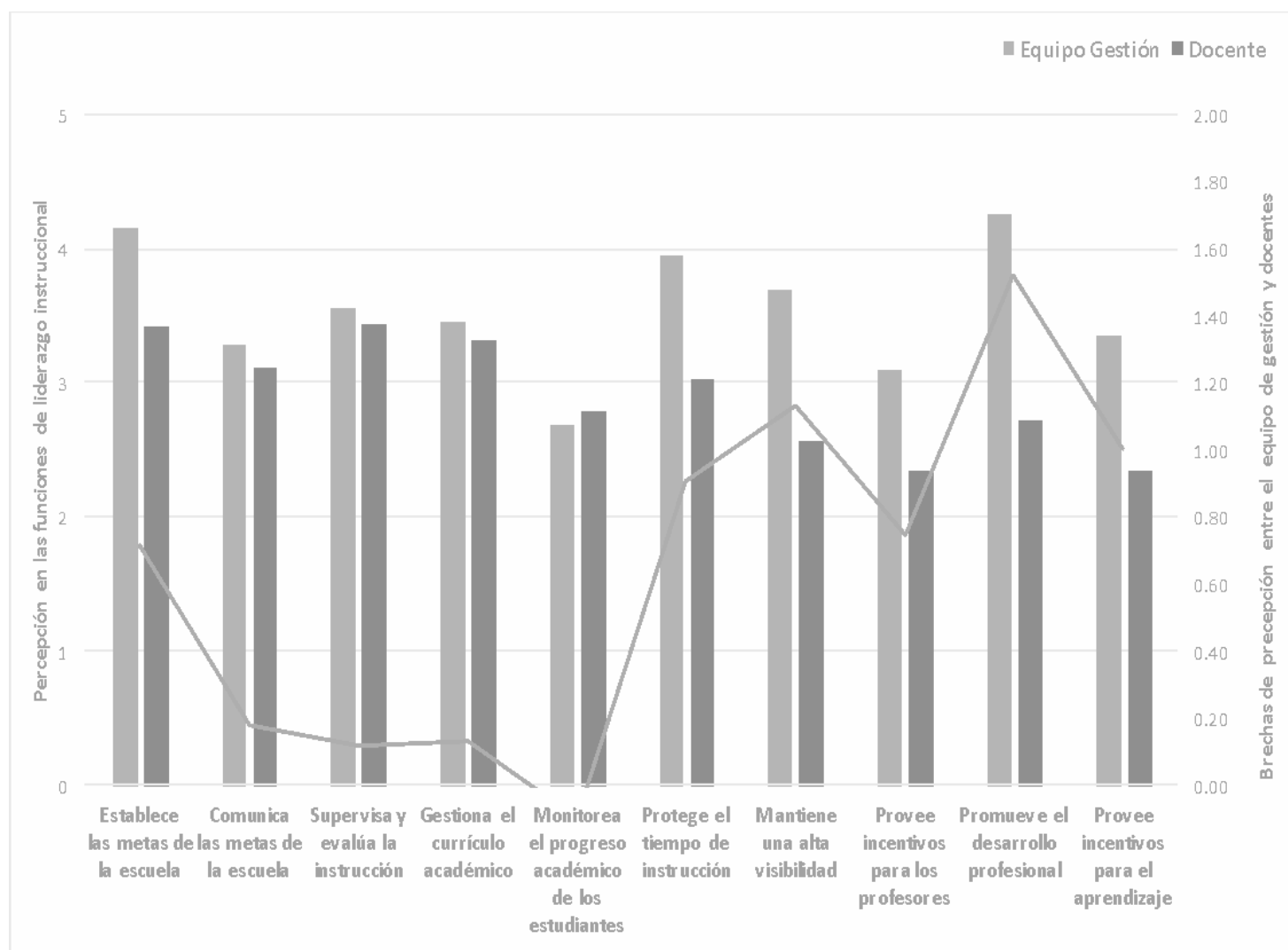

Figura 2. Resultados PIMRS Colegio 1, mayo 2017. Fuente: Elaboración propia.

La evidencia levantada a través del instrumento sobre desarrollo de liderazgo instruccional fue confirmada por los directivos, quienes explicaban estas diferencias en base a la dedicación de su labor principalmente a tareas administrativas y no exclusivamente pedagógicas. Considerando esto, en conjunto con el equipo directivo se determinó trabajar en las siguientes acciones: a) Comunica las metas de la escuela, b) Provee incentivos para profesores y c) Provee incentivos para el aprendizaje.

El método de trabajo establecido consideró la teoría de implementación de las CPA para atender a las necesidades específicas asociadas a las dimensiones del Liderazgo Instruccional consideradas relevantes para los participantes del Colegio 1. En específico, se trabajó junto al equipo directivo en reuniones periódicas desarrolladas en el propio colegio, para analizar los datos, revisar la propuesta del plan, incorporar sugerencias propias al contexto, analizar su implementación y resolver dudas o problemáticas que emergieran en el proceso. 
A continuación, se resume en la Tabla 1 la propuesta de trabajo elaborada entre los investigadores y participantes del equipo directivo.

Tabla 1

Síntesis de acciones según los focos de las comunidades profesionales de aprendizaje y cuestionario PIMRS de Liderazgo Instruccional elaborada junto a directivos Colegio 1.

\begin{tabular}{|c|c|c|c|c|c|}
\hline $\begin{array}{l}\text { Dimensión y } \\
\text { acción PIMRS }\end{array}$ & Foco CPA & Acciones & Frecuencia & Involucrados & $\begin{array}{l}\text { Evaluación de los } \\
\text { profesores }\end{array}$ \\
\hline \multirow{2}{*}{$\begin{array}{l}\text { II. Define la } \\
\text { misión de la } \\
\text { escuela: } \\
\text { Comunica las } \\
\text { metas } \\
\text { escolares }\end{array}$} & \multirow{2}{*}{$\begin{array}{l}\text { Compartir } \\
\text { misión y } \\
\text { visión de la } \\
\text { escuela }\end{array}$} & $\begin{array}{l}\text { Cada curso analiza y } \\
\text { difunde en su aula la } \\
\text { misión y visión del } \\
\text { colegio junto a su } \\
\text { profesor tutor }\end{array}$ & $\begin{array}{l}\text { Una vez por } \\
\text { semestre }\end{array}$ & $\begin{array}{l}\text { Directivos, } \\
\text { profesores- } \\
\text { tutores, } \\
\text { apoderados, } \\
\text { estudiantes }\end{array}$ & $\begin{array}{l}\text { Percepción de la } \\
\text { comunidad de las } \\
\text { actividades } \\
\text { realizadas }\end{array}$ \\
\hline & & $\begin{array}{l}\text { Apoya a profesores a } \\
\text { reconocer y difundir } \\
\text { logros de estudiantes } \\
\text { y su vinculación con } \\
\text { desarrollo misión y } \\
\text { visión del colegio }\end{array}$ & $\begin{array}{l}\text { Una vez por } \\
\text { semestre }\end{array}$ & $\begin{array}{l}\text { Directivos, } \\
\text { profesores } \\
\text { tutores, } \\
\text { apoderados, } \\
\text { estudiantes }\end{array}$ & $\begin{array}{l}\text { Análisis de las } \\
\text { implicancias del } \\
\text { reconocimiento de } \\
\text { los logros y su } \\
\text { vínculo con la } \\
\text { misión del colegio }\end{array}$ \\
\hline \multirow{3}{*}{$\begin{array}{l}\text { III. Fomenta } \\
\text { un clima } \\
\text { adecuado para } \\
\text { el aprendizaje: } \\
\text { Provee } \\
\text { incentivos } \\
\text { para los } \\
\text { profesores }\end{array}$} & $\begin{array}{l}\text { Foco grupal } \\
\text { en } \\
\text { aprendizaje } \\
\text { de los } \\
\text { estudiantes: } \\
\text { resultados y } \\
\text { motivación }\end{array}$ & $\begin{array}{l}\text { Reforzar y reconocer } \\
\text { desempeños } \\
\text { destacados: felicitar } \\
\text { públicamente a } \\
\text { profesores que } \\
\text { destacaron en el logro } \\
\text { de los objetivos de } \\
\text { cada curso o por } \\
\text { algún logro particular }\end{array}$ & $\begin{array}{l}\text { Al inicio de } \\
\text { cada semestre }\end{array}$ & $\begin{array}{l}\text { Jefe } \\
\text { pedagógico }\end{array}$ & $\begin{array}{l}\text { Impresiones y } \\
\text { satisfacción con la } \\
\text { medida de los } \\
\text { profesores } \\
\text { implicados }\end{array}$ \\
\hline & \multirow{2}{*}{$\begin{array}{l}\text { Apertura a } \\
\text { la mejora } \\
\text { en base a } \\
\text { prácticas } \\
\text { exitosas }\end{array}$} & $\begin{array}{l}\text { Celebra logros del } \\
\text { establecimiento: } \\
\text { actos, juegos de } \\
\text { esparcimiento }\end{array}$ & Trimestral & $\begin{array}{l}\text { Equipo } \\
\text { Directivo }\end{array}$ & $\begin{array}{l}\text { Impresiones } \\
\text { comunidad escolar }\end{array}$ \\
\hline & & $\begin{array}{l}\text { Organiza reuniones } \\
\text { de difusión de } \\
\text { técnicas de } \\
\text { aprendizajes } \\
\text { desarrolladas por } \\
\text { docentes de la escuela }\end{array}$ & Trimestral & $\begin{array}{l}\text { Jefe } \\
\text { pedagógico }\end{array}$ & $\begin{array}{l}\text { Evaluación verbal o } \\
\text { escrita de } \\
\text { profesores }\end{array}$ \\
\hline
\end{tabular}

Fuente: Elaboración propia. 
Las reuniones mensuales entre el equipo directivo y el equipo de investigación, desarrolladas durante el segundo semestre del año 2017, permitieron monitorear y orientar el progreso de cada acción de acuerdo con el foco correspondiente. Estas reuniones fueron registradas a través de notas de campo, en un proceso sistemático y consistente que dio paso a la siguiente etapa.

\section{Etapa III. Evaluación del alcance de la propuesta trabajada}

La Etapa III se inició al finalizar el año escolar, período en el cual se realizó la evaluación cuantitativa por medio de la reaplicación de los ítems del cuestionario PIMRS asociados a las acciones iniciadas. En la Tabla 2 se presentan resultados para las tres funciones definidas en el PIMRS que se trabajaron durante el período de investigación.

Tabla 2

Resultados pre y post cuestionario PIMRS, Colegio 1.

\begin{tabular}{lcccc}
\hline & \multicolumn{2}{c}{ Equipo de gestión } & \multicolumn{2}{c}{ Docentes } \\
\hline $\begin{array}{l}\text { Funciones directivas } \\
\text { incluidas en la intervención }\end{array}$ & Antes & Después & Antes & Después \\
\hline $\begin{array}{l}\text { Comunica las metas } \\
\text { escolares }\end{array}$ & M 3.4 & M 4.1 & M 3.1 & M 3.3 \\
\hline $\begin{array}{l}\text { Provee incentivos para } \\
\text { profesores }\end{array}$ & DT 1.51 & DT 1.10 & DT 1.19 & DT 1.19 \\
\hline $\begin{array}{l}\text { Provee incentivos para el } \\
\text { aprendizaje }\end{array}$ & DT 0.74 & DT 0.85 & DT 1.16 & DT 1.24 \\
\hline
\end{tabular}

Fuente: Elaboración propia

En todos los casos, al comparar los resultados al inicio y término de la investigación, se aprecia que existe un aumento en la percepción por parte del equipo directivo y los profesores en términos del grado de implementación de las tres funciones de liderazgo instruccional definidas en el PIMRS y abordadas en el proceso de innovación de la gestión del equipo de gestión del Colegio 1. Sin embargo, la diferencia en percepción del equipo directivo antes y después no 
representa cambios significativos: $\mathrm{F}(1,68)=2.50, p=.12$. En cambio, la diferencia en la percepción de los docentes sí presenta cambios significativos: $\mathrm{F}(1,589)=10.62, p<.01, \mid=.02$.

Junto a la reaplicación del cuestionario se desarrollaron entrevistas semiestructuradas (Vieytes, 2004) a los miembros del equipo directivo. Las entrevistas fueron analizadas a través del método de análisis de contenido (Gibbs, 2012). Este procedimiento, sostenido en la reducción de la información, primero a través de códigos y luego en categorías con sus respectivas subcategorías, en este caso fueron emergentes, es decir se formaron a partir del relato de los participantes y no desde la teoría que fundamenta el estudio. Las dos categorías que permitieron comprender las implicancias del plan de trabajo, más allá de los datos cuantitativos, fueron:

Categoría Autoestima académica de los profesores: Esta categoría se comprende como la relevancia que cobra la valoración de los docentes de la institución educativa sobre sus propias capacidades para mejorar los aprendizajes de sus estudiantes a través del cambio y la innovación. Para los directivos, la experiencia del plan de trabajo permitió organizar actividades en los cuales los docentes evidenciaran sus actividades pedagógicas frente a sus pares, dando así importancia a los mecanismos de reconocimiento de logros profesionales de los docentes. El desarrollo de las ideas permitió la generación de dos subcategorías:

Subcategoría Incentivos y reconocimientos: Los miembros del equipo directivo relatan que la experiencia de generar incentivos para los profesores, especialmente de reconocer sus esfuerzos y capacidades, es una estrategia novedosa que genera un ambiente distinto para el trabajo diario. Así, si bien dieron cuenta de que el trabajo del profesorado no había sido relacionado con este tipo de estrategias - “A nosotros nunca nos han reconocido nada, sabemos que tenemos que trabajar bien y listo" (ED 1) — reconocen que se dedicaron a organizar un modo de estimular a los docentes:

Realizamos en el último consejo un reconocimiento de los profesores y administrativos, los citamos a un consejo formal y se destacaron cualidades humanas y profesionales. Hicimos dos categorías una de personas que tienen un desempeño en ámbitos administrativos y a profesores que se habían destacado a nivel profesional (ED 2).

Este esfuerzo les significó una mirada crítica y relevante a las tareas que pueden hacer como directivos y el impacto que tiene en los profesores.

Los directivos evidenciaron que la innovación en el ámbito del reconocimiento al trabajo docente era un aporte al bienestar cotidiano de los profesores y demás miembros de la comunidad 
educativa. Además, observaron que era un espacio que daba sostenibilidad a la mejora permanente del profesorado, como se evidencia en las siguientes citas: "Los docentes tienen mayor compromiso y participación a partir del reconocimiento, hay una mejor disposición para trabajar aún en horarios no recurrentes" (ED 1); "Pudimos tomar conciencia del valor de felicitar el trabajo del profesor, de acompañar sus reflexiones sobre lo que está haciendo. Hicimos una hoja de acuerdos donde también se les felicita por escrito" (ED 2).

Lo anterior revela que el equipo directivo logró percibir que era posible, desde su propia iniciativa, fortalecer el desarrollo docente y alcanzar mejoras, incluso inesperadas: “Los profesores nuevos se integran a un modelo más cooperativo y eso da funcionalidad a la innovación, hay un ambiente de trabajo más positivo" (ED 2).

Subcategoría Aceptación de desafíos: Un concepto recurrente entre los entrevistados fue el de "desafío". Dieron cuenta de que los profesores tenían una mirada abierta al cambio e innovación que les supusiera participar y mejorar el desarrollo de la docencia. Como relataron, la experiencia fue un "desafío de hacer visible la práctica pedagógica y los que participaron presentaron mayor seguridad en sí mismos y se vieron cambios hacia el 2018” (ED 1).

Se observa en los directivos una mirada reflexiva respecto a los profesores, una mirada incluso de sorpresa frente a las respuestas, en tanto la experiencia superó sus expectativas y debieron adecuar los espacios y tiempo a la participación generada, como se evidencia en esta respuesta: "A los profesores, mostrar sus prácticas les ayudó en su autoestima profesional, pensamos que serían dos, pero fueron más y traían material fotocopiado para compartir con sus compañeros" (ED 2).

De este modo, los directivos pudieron vivenciar la importancia de compartir experiencias pedagógicas como un modo de potenciar el desarrollo de los profesores, pero además aprender, como equipo directivo, a conocer a los profesores que forman parte del colegio. Así la autoestima académica del docente se relacionó con la aceptación de desafíos profesionales que permitieran compartir su saber con los pares.

Categoría Equipos directivos acompañados de reflexión académica: El otro gran tema emergente de las entrevistas fue el proceso de acompañamiento como un espacio de formación y generación de capacidades directivas. Al respecto los directivos dieron cuenta de que en muchos sentidos el plan de trabajo correspondió a un proceso que les exigió salir de lo cotidiano y revisar 
sus propios modos de ser y hacer su trabajo, pero en compañía. Las subcategorías que emergieron de este significado de la experiencia fueron:

Subcategoría Reflexión sobre las propias prácticas: Los directivos abordaron la importancia dada al proceso de innovación como fuente de reflexión para sus encuentros habituales a nivel de equipo directivo. Los resultados entregados en reuniones con el equipo de investigación y la propuesta de trabajo generada les permitió a ellos mismos reenfocar los ámbitos de trabajo del equipo de gestión hacia perspectivas que ya habían intuido, pero no se atrevían a desarrollar.

El equipo directivo se ha fortalecido en compartir logros y desafios, incluir a más personas, aceptar propuestas más que dar direcciones. Se han buscado mecanismos para informar y para generar instancias de propuestas (ED 2).

El plan innovador ayudó a visibilizar el tema del liderazgo, que debe ser distribuido y no unipersonal, antes habíamos visto este punto, pero con el plan salimos de la mirada hacia la funcionalidad, le dimos a una estructura una materialización (ED1)

Este proceso tuvo implicancias diferentes para cada uno de los miembros, pero en los dos casos fueron alcances relevantes que les ayudaron a realizar sus tareas cotidianas de modo reflexivo y a proyectar un camino de innovación.

Subcategoría Acompañamiento académico: Esta subcategoría aborda el significado atribuido a la experiencia de ser acompañados por un equipo de investigación universitario. Para los directivos entrevistados el modo de hacer el acompañamiento fue relevante y decisivo para continuar cada etapa del proceso. Los implicados se refieren a una forma dialogante, que reconoce la experiencia y acompaña sin imponer transformaciones. Además, le dan una profunda importancia a que el equipo buscara adecuar modelos al contexto sin forzar las aplicaciones.

La mirada externa del equipo de investigación ayudó a mirar elementos que vemos todos los días pero que no hacíamos nada por cambiar. Las ATE anteriormente nos habían dicho hay que hacer, pero trabajar en colaboración con ustedes ha sido distinto porque se han conversado los resultados, han presentado lo esperable en colegios similares y se ha analizado, entonces hemos podido darnos cuenta, tomar conciencia de los errores y ver las potencialidades que tenemos a nivel interno también (ED 2).

Subcategoría Alcances del plan: El proceso innovador permitió obtener resultados satisfactorios desde la experiencia de los directivos, tanto en construir un ambiente de trabajo coherente y desafiante como en involucrar a más miembros de la comunidad en el trabajo habitual. 
Los directivos se abrieron a buscar modos diferentes para alcanzar sus metas, como explican a continuación:

"Hacer equipos y crear cambios hizo que quienes no estaban en esa sintonía se fueran del colegio, porque se dieron cuenta que no estaban en sintonía” (ED 2).

"Nos ha ayudado a correr la barrera de que las peticiones del equipo directivo son exigencias, para comprender que se está buscando que los niños aprendan. Trabajar la misión, la visión, el sello nos ayudó a crear un ambiente de mayor compromiso, de estudiantes por ejemplo los centros de estudiantes y los profesores" (ED 2).

Otro elemento importante de acuerdo con su percepción fue dar herramientas que tuvieran continuidad y desafíos:

"Quedó establecido en el calendario que sea un tema dentro de los consejos para continuar con el trabajo realizado, pero considerando el contexto teórico y pidiéndole al profesor que nos ayude a desarrollar las innovaciones con otros profesores" (ED 1).

"El plan nos ha dado herramientas como lo del reconocimiento. Lo que hicimos quedó como un hito histórico, pero necesitamos mejorar eso mismo para los estudiantes, para darle cabida a la autoestima académica y la formación integral, estos conceptos no pueden ser solamente de pasillos, tiene que ser formal" (ED 2).

\section{Discusión y conclusiones}

El trabajo se inició bajo el alero de un Plan que atiende a lo que hace tiempo se ha indicado como un factor relevante del desarrollo educativo latinoamericano, que "el ámbito académico se aboque a formar jóvenes académicos e investigadores cuya perspectiva educativa esté basada en contexto latinoamericanos locales y no en experiencias remotas y 'ajenas"' (Oplatka citado en Weinsten, 2017, p. 255).

Esta perspectiva abre puertas a comprender la gestión en contextos locales como un espacio de desarrollo de modelos que reconocen las particularidades de cada cultura y que, por ende, respetan las narrativas y contextos sociales. Este es un aspecto que, como se evidenció en este caso, impulsa cambio educativo desde elementos significativos para los implicados. Por este motivo, realizar la investigación sobre una temática relevante para los colegios, especialmente para el Colegio 1, resultó un desafío alentador para este grupo de investigación. La investigación en el ámbito nacional ha descrito que en Chile, como evidencian Weinstein \& Muñoz (2012), el director 
se dedica principalmente a tareas administrativas y de representación política de la escuela por sobre tareas asociadas al liderazgo pedagógico. Estas últimas habitualmente las desarrolla el jefe de la UTP, quien no siempre cumple un rol de liderazgo profesional, sino uno técnico y burocrático.

Junto a lo mencionado, y en el contexto del estudio, el Plan fue una experiencia relevante de acompañamiento, orientación al cambio y apoyo en procesos de alta exigencia para el equipo de gestión. Esto se condice por ejemplo en la aplicación del post-test, proceso en que, si bien no se detectan cambios en la autopercepción directiva, sí fue relevante la evidencia manifestada por los docentes, que muestran una acción directiva más intencionada y por tanto más efectiva. Estos elementos, de acuerdo con García-Martínez, Higueras-Rodríguez y Martínez-Valdivia (2018), serían un aporte de la propuesta realizada al desarrollo de un problema ampliamente descrito, consistente en la rígida estructura de liderazgo de las instituciones educativas. Esta es una situación que limita la posibilidad de cambio y la capacidad de dar respuesta las demandas locales y nacionales.

El proceso de trabajo, desde la etapa de diagnóstico hasta la de evaluación, permitió vivenciar la relevancia del desarrollo de capacidades in situ para directivos y profesores, lo cual supone un método efectivo para generar cambios sostenibles (Hargreaves y Fullan, 2014). En tiempos en los que el cambio y la medición del desarrollo profesional de los docentes es un insumo frecuente pero ajeno a la realidad, se vuelve fundamental establecer diálogos que conecten los procesos informales de aprendizaje en el trabajo con orientaciones estratégicas coherentes (Fullan, 2012).

Aportes como los descritos se generaron en tanto la experiencia de diálogo entre la academia y la escuela abrió posibilidades no previstas dentro de esta investigación, como fue atender a necesidades emergentes de grupos específicos del Colegio 1, quienes frente a la incorporación de nuevos docentes al colegio desarrollaron estrategias en el marco del Plan. De este modo, los directivos, por medio de los insumos construidos en este diálogo, pudieron establecer puentes para facilitar un mejor tránsito y crecimiento profesional en el colegio.

En consecuencia, el diálogo enriqueció la reflexión a la vez que la gestión, lo que puede aportar a generar fines más complejos, validando ampliamente la generación de investigación situada y con ello los aprendizajes informales. Este punto, como han descrito Kyndt, Gijbels, Grosemans y Donche (2016), se relaciona con constantes procesos de aprendizajes en instancias 
no registradas y por tanto que no pueden ser seguidas en sus implicancias. Sin embargo, el caso implementado fue posible de seguir, reorientar y apoyar cuando fue necesario, mejorando con ello la calidad del trabajo directivo.

La investigación realizada demanda revisar sus limitaciones y proyecciones, las cuales transitan entre los desafíos que supone para los equipos directivos —altamente exigidos por la legislación nacional, especialmente en tareas administrativas - el dar continuidad a procesos de cambio. Esta es una tarea que les genera dificultades y desafíos frente a la relevancia que los mismos equipos le dan a la reflexión que se genera a través de la participación en planes como el implementado.

Los implicados evidenciaron que las temáticas y procesos del Plan fueron un espacio de desafío en el día a día, por ejemplo, respecto al valor de la misión-visión del Colegio. Esto confiere a las universidades la demanda de continuar con dinámicas de seguimiento del quehacer del equipo directivo para acompañar e incentivar la focalización de la innovación y así evitar las prácticas fragmentadas (Fullan, 2012).

Por otra parte, se evidencia que, existiendo este tipo de redes y la disposición requerida para revisar sus prácticas de liderazgo, así como para buscar estrategias de mejora acompañados por equipos de investigación, es posible y altamente valioso para ambos grupos establecer diálogos y metodologías que resulten innovadoras. En definitiva, se trata de actos de apertura hacia enfoques de corresponsabilidad frente a los fines de la educación en nuestros tiempos.

\section{Referencias}

Ahumada, L., Galdames, S., \& Clarke, S. (2016). Understanding leadership in schools facing challenging circumstances: a Chilean case study. International Journal of Leadership in Education, 19(3), $264-279$. http://doi.org/10.1080/13603124.2015.1048744

Aravena F., \& Hallinger, P. (2018). Systematic review of research on educational leadership and management in Latin America, 1991-2017. Educational Management Administration \& Leadership, 46(2), 207-225. https://doi.org/10.1177/1741143217745882

Coffey, A., y Atkinson, P. (2003). Encontrar el sentido a los datos cualitativos. Estrategias complementarias de investigación. Antioquia, Colombia: Editorial Universidad de Antioquia.

Creswell, J. (2012). Educational research: Planning, conducting, and evaluating quantitative and qualitative research (4th ed.). Boston, MA: Pearson.

Cuellar, C., \& Giles, D. L. (2012). Ethical practice: a study of Chilean school leaders. Journal of Educational Administration, 50(4), 420-436. http://doi.org/10.1108/09578231211238576 
Darling-Hammond, L., Burns, D., Campbell, C., Lin Goodwin, A., Hammerness, K. Low, E-L., McIntyre, A.... Zeichner, K. (2017). Empowered Educators: How high performing systems shape teaching quality around the world. San Francisco, CA: Wiley/Jossey Bass.

Day, C., Sammons, P., Hopkins, D., Harris, A., Leithwood, K., Gu, Q., Brown, E.... Kingston, A. (2009). The Impact of School Leadership on Pupil Outcomes. Final Report (No. DCSF-RR108). Department for children, schools and families. Recuperado de https://dera.ioe.ac.uk/11329/1/DCSF-RR108.pdf

Falabella, A., y de la Vega, L. (2016). Políticas de responsabilización por desempeño escolar: Un debate a partir de la literatura internacional y el caso chileno. Estudios pedagógicos (Valdivia), 42(2), 395-413. https://doi.org/10.4067/S0718-07052016000200023

Flessa, J. (2014). Learning from School Leadership in Chile. Canadian and International Education, 43(1), Article 2. Recuperado de http://ir.lib.uwo.ca/cie-eci/vol43/iss1/2

Flores, C. (2014). Inducción de profesores novatos en Chile: un estudio de caso. Pensamiento Educativo: Revista de Investigación Educacional Latinoamericana, 51(2), 41-55. Https://doi.org/10.7764/PEL.51.2.2014.4

Fromm, G., Olbrich, I., y Volante, P. (2015). Fidelidad de la implementación de prácticas de liderazgo instruccional. Magis, Revista Internacional de Investigación en Educación, 7(15), 117-134.

Fullan, M. (2012). Los nuevos significados del cambio en la educación. Barcelona, España: Octaedro.

García-Martínez, I., Higueras-Rodríguez, L., y Martínez-Valdivia, E. (2018). Hacia la Implantación de Comunidades Profesionales de Aprendizaje Mediante un Liderazgo Distribuido. Una Revisión Sistemática. REICE. Revista Iberoamericana sobre Calidad, Eficacia y Cambio en Educación, 16(2), 117-132. https://doi.org/10.15366/reice2018.16.2.007

Gibbs, G. (2012). El análisis de Datos Cualitativos en Investigación Cualitativa. Madrid, España: Morata.

Hallinger, P. (2011). Leadership for learning: lessons from 40 years of empirical research. Journal of Educational Administration, 49(2), 125-142. http://doi.org/10.1108/09578231111116699

Hallinger, P. (2019). Science mapping the knowledge base on educational leadership and management from the emerging regions of Asia, Africa and Latin America. 1965-2018. Educational Management Administration and Leadership, 48(2), 209-230. https://doi.org/10.1177/1741143218822772

Hallinger, P., \& Murphy, J. (1985). Assessing the Instructional Management Behavior of Principals. The Elementary School Journal, 86(2), 217. http://doi.org/10.1086/461445

Hargreaves, A., \& Fullan, M. (2014). Capital Profesional. Madrid, España: Morata.

Hargreaves, A., \& Shirley, D. (2012). The global fourth way: The quest for educational excellence. Thousand Oaks, CA: Corwin Press.

Harris, A. (2012). Liderazgo y Desarrollo de capacidades en la escuela. Santiago, Chile: Fundación Chile- CAP.

Horn, A., y Marfan, J. (2010). Relación entre liderazgo educativo y desempeño escolar: Revisión de la investigación en chile. Psicoperspectivas, 9(2), 82-104. Recuperado de https://scielo.conicyt.cl/pdf/psicop/v9n2/art05.pdf

Kyndt, E., Gijbels, D., Grosemans, I., y Donche, V. (2016). Teachers' Everyday Professional Development: Mapping Informal Learning Activities, Antecedents, and Learning Outcomes. Review of Educational Research, 86, 1111-1150. https://doi.org/10.3102/0034654315627864 
Klieger, A. (2016). Principals and teachers: Different perceptions of large-scale assessment. International Journal of Educational Research, 75, 134-145. http://doi.org/10.1016/j.ijer.2015.11.006

Ley N $\mathrm{N}^{\circ}$ 20.903. Crea el Sistema de Desarrollo Profesional Docente y modifica otras normas, 01 abril 2016.

Ley $\mathrm{N}^{\circ}$ 20.845. De Inclusión Escolar que regula la admisión de los y las estudiantes, elimina el financiamiento compartido y prohíbe el lucro en establecimientos educacionales que reciben aportes del estado, 08 junio 2015.

Ley N ${ }^{\circ}$ 20.248. Establece Ley de Subvención Escolar Preferencial, 01 de febrero de 2008.

López A, P., y Gallegos A, V. (2014). Prácticas de liderazgo y el rol mediador de la eficacia colectiva en la satisfacción laboral de los docentes. Estudios Pedagógicos (Valdivia), 40(1), 163-178. http://doi.org/10.4067/S071807052014000100010

Marradi, A., Archenti, N., y Piovani, J. (2018). Manual de metodología de las ciencias sociales. Buenos Aires, Argentina: Siglo XXI.

Ministerio de Educación de Chile. (2015). Marco para la Buena Dirección y Liderazgo Escolar: Documento de trabajo. Recuperado de https://liderazgoescolar.mineduc.cl/wpcontent/uploads/sites/55/2016/04/MBDLE_2015.pdf

Muñoz Martínez, M., \& Garay Garay, F. (2015). La investigación como forma de desarrollo profesional docente: Retos y perspectivas. Estudios Pedagógicos (Valdivia), 41(2), 389-399. http://doi.org/10.4067/S071807052015000200023

Oplatka, I. (2019). El surgimiento de la gestión educativa como campo de estudio en América Latina. En J. Weinstein (ed), Liderazgo educativo en la escuela: nueve miradas (pp. 253-276). Santiago, Chile: Ediciones Universidad Diego Portales. Recuperado de http://cedle.cl/wp-content/uploads/2018/03/LiderazgoEducativo-en-la-Escuela.-Nueve-miradas.pdf

Organisation for Economic Cooperation and Development. (2019). TALIS 2018 Results (Volume I): Teachers and School Leaders as Lifelong Learners. París, Francia: OECD Publishing.

Quiroga, M., y Aravena, F. (2018). ¿Qué tipos de datos recolectan los directores? Consecuencias para la elaboración de planes de mjejora. Páginas de Educación, 11(2), 24-39.

Roberts, S., y Pruitt, E. (2009). Schools as Professional Learning Communities. California, CA: Corwin Press.

Ruffinelli Vargas, A. (2016). Ley de desarrollo profesional docente en Chile: de la precarización sistemática a los logros, avances y desafíos pendientes para la profesionalización. Estudios pedagógicos (Valdivia), 42(4), 261-279. https://doi.org/10.4067/S0718-07052016000500015

Sepúlveda, F., y Aparicio Molina, C. (2019). Habilidades de liderazgo instruccional de directores y sus equipos de gestión de escuelas de la región del Biobío, Chile: fortalezas y desafíos. Revista De Investigación Educativa, 37(2), 487-503. https://doi.org/10.6018/rie.37.2.329861

Tapia-Gutiérrez, C., Becerra-Peña, S., Mansilla-Sepúlveda, J., y Saavedra-Muñoz, J. (2011). Liderazgo de los directivos docentes en contextos vulnerables. Educación y Educadores. Recuperado de http://educacionyeducadores.unisabana.edu.co/index.php/eye/article/view/1930/2517 
Tanriogen, A. ., \& Iscan, S. (2016). Effect of distributive leadership behaviours of foreign language schools principals on the job satisfaction of instructors. International Journal of Educational Administration and Policy Studies. 8(6), 48-56. http://doi.org/10.5897/IJEAPS2016.0472

Ulloa Garrido, J., Nail Kröyer, O., Castro Hidalgo, A., y Muñoz Reyes, M. (2012). Problemas de gestión asociados al liderazgo como función directiva. Estudios Pedagógicos (Valdivia), 38(1), 121-129. http://doi.org/10.4067/S0718-07052012000100007

Valenzuela, J. P., y Montecinos, C. (2017). Structural reforms and equity in Chilean schools. Oxford Research Encyclopedia of Education. http://doi.org/10.1093/ACREFORE/9780190264093.013.108

Vieytes, R. (2004). Metodología de la investigación en organizaciones, mercado y sociedad: epistemología y técnicas. Buenos Aires, Argentina: Editorial de las Ciencias.

Weinstein, J., y Hernández Vejar, M. (2014). Políticas hacia el liderazgo directivo escolar en Chile: Una mirada comparada con otros sistemas escolares de América Latina. Psicoperspectivas. Individuo y Sociedad, 13(3), 52-68. https://doi.org/10.5027/psicoperspectivas-Vol13-Issue3-fulltext-468

Weinstein, J., y Muñoz, G. (2012). ¿Qué sabemos de los directores de escuela en Chile? Santiago, Chile: CEPPE y Centro de Innovación en Educación Fundación Chile.

Weinstein J. (Ed.). (2017). Liderazgo educativo en la escuela: nueve miradas. Santiago, Chile: Ediciones Universidad Diego Portales. Recuperado de http://cedle.cl/wp-content/uploads/2018/03/Liderazgo-Educativo-en-laEscuela.-Nueve-miradas.pdf

Weinstein, J., Muñoz, D., Sembler, M., y Marfán, J. (2019). Una década de investigación empírica sobre el liderazgo educativo en Chile. Una revisión sistemática de los artículos publicados en revistas indexadas (2008-2019). Calidad en la Educación, 51, 15-52. https://doi.org/10.31619/caledu.n51.671

Yin, R. (1993). Applications of case study research. London, United Kingdom: Sage.

\section{Contribución autoral}

a) Concepción y diseño del trabajo; b) Adquisición de datos; c) Análisis e interpretación de datos; d) Redacción del manuscrito; e) revisión crítica del manuscrito.

C.A.M. ha contribuido en a, b, c, d, e.

F.S.L. ha contribuido en a, b, c, e.

X.V.H. ha contribuido en b, c, e.

V.C.M. ha contribuido en c, e.

G.C.S. ha contribuido en d, e.

M.V.R. ha contribuido en $\mathrm{d}$, e.

\section{Editora científica responsable}

Mag. Florencia Deleon 